\title{
Realization of a Device for the Evaluation of the Muscular Effort through the Electromyogram Signal Emg
}

\author{
F. Medjahed ${ }^{1}$, A. Mokdad ${ }^{1}$, S.M Debbal ${ }^{1 *}$ \\ ${ }^{1}$ Genie Biomedical Laboratory (GBM), Faculty of Technology, University of Abou.Bakr.Belkaid, Tlemcen, Algeria. \\ *Corresponding author: S.M Debbal, Genie Biomedical Laboratory (GBM), Faculty of Technology, University of Abou.Bakr.Belkaid, \\ Tlemcen, Algeria.
}

Received date: May 05, 2021; Accepted date: May 12, 2021; Published date: May 20, 2021

Citation: F. Medjahed, A. Mokdad, S.M Debbal, (2021). Realization of a Device for the Evaluation of the Muscular Effort through the Electromyogram Signal Emg. J Clinical Research and Reports, 8(1); DOI:10.31579/2690-1919/169

Copyright: (C) 2021 S.M Debbal. This is an open access article distributed under the Creative Commons Attribution License, which permits unrestricted use, distribution, and reproduction in any medium, provided the original work is properly cited.

\begin{abstract}
In this work, a device for the evaluation of the muscular effort through the electromyogram signal is produced. This device consists essentially of three parts: the sensor part, the shaping portion, the acquisition part and the software part. The sensor part allows the EMG signal to be collected by means of surface electrode. The shaping port is realized based on an instrumentation amplifier. The acquisition part concerns the analogue digital conversion and the transfer of the digital data to the pc; this is done via an arduino card, which is equipped with a microcontroller for the visualization in real time and the storage of the EMG signal on the pc on which the processing logitiels will be implemented. The signal thus processed must be displayed with the data allowing the evaluation of the effort on the monitor of the pc through a graphical interface; these are the different steps that are carried out to finalize this work

Key words: Electromyogram (EMG), surface electrodes, instrumentation amplifier, arduino card; PC; matlab; frequency
\end{abstract}

\section{Introduction}

In order for movement to occur, the muscles, bones, tendons and ligaments must all work together in response to signals from the brain [1].

The musculoskeletal system, consisting of muscles, bones and joints, requires special attention and care; otherwise stiffness, stiffness, contractures, pain and inflammation will become the companions of illadapted and poorly prepared physical activity had not the opportunity to feel the painful consequences of an unusual gesture. To help the doctor to assert the existence or not of a muscular pain, the electromyography is particularly useful in the assessment of certain muscular deficits in order to evaluate this muscular effort. This is the goal of this project of end of studies. In fact, the goal is to make a device that allows the detection of the signal Electromyogram (EMG), its shaping and its treatment to assess the muscular effort.

Electromyography is the study of muscle function through the inquiry of the electrical signal the muscles emanate [2]. This signal is detected through electrodes that must be properly arranged on a muscle. However, the signal detected is very low level and embedded in different kinds of noise, it is necessary to amplify and filter it to be exploited. However, and in order to evaluate the effort through this signal, digital processing is necessary. To carry out this processing, it is essential to scan it and transfer it to a PC on which the processing software will be implemented. The signal thus processed must be displayed with the data enabling the evaluation of the effort on the monitor of the PC through a graphical interface. These are the different steps that must be carried out to finalize this device.

\section{Method}

\section{Acquisition of EMG signal:}

The EMG signal once detected and formatted is digitized for transmission to the $\mathrm{PC}$ for digital processing and display. The card used for this scan and interface with the PC is the ARDUINO-UNO card. However, since the analog inputs of the card accept only unipolar signals, an offset circuit has been realized. This circuit shifts the EMG signal between $0 \mathrm{~V}$ and a maximum of $5 \mathrm{~V}$ [3]. Open source ARDUINO software has been formatted to allow acquisition of the EMG signal. The key in this formatting of the PC acquisition and interface software is the choice of the input port, and the frequency and sampling. The selected port is port A0; however, the sampling frequency is chosen according to the maximum frequency of the EMG signal which is about $500 \mathrm{~Hz}$. The frequency is then fixed at $1000 \mathrm{~Hz}$ [4]. (Figure 1) below shows the picture of the device made. 


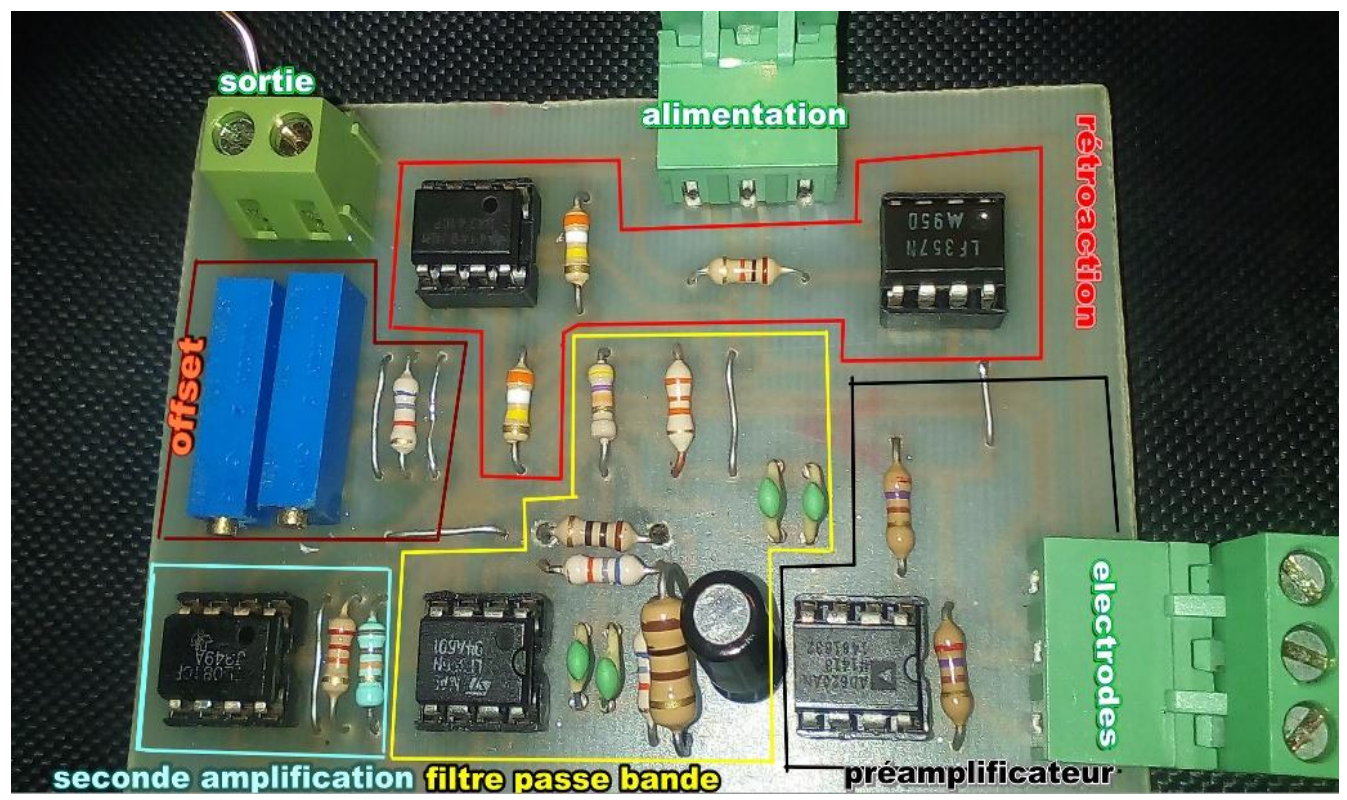

Figure 1. Photo of the realized device.

\section{Acquisition of the EMG signal through the Arduino acquisition board:}

The EMG signal thus obtained is applied to the Arduino acquisition board via the analog input A0 to be digitized and transferred to the PC for digital processing. (Figure 2) shows a picture of the realized device connected to the PC.

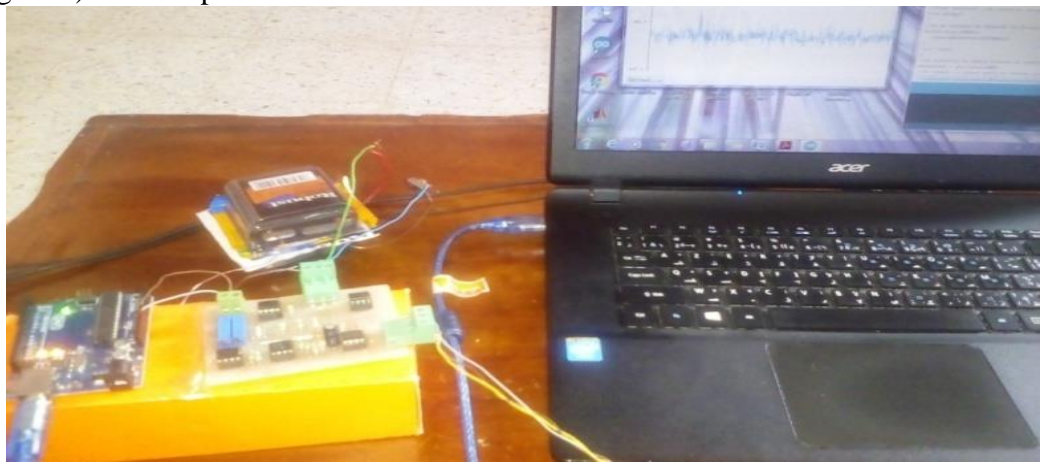

Figure 2. Photo representing the device connected to the ARDUINO card and the PC

The EMG signal thus digitized and transferred to the PC or it can be viewed on the serial monitor of the Arduino IDE [5] as values or on serial plotter as a graph (see figure 3)

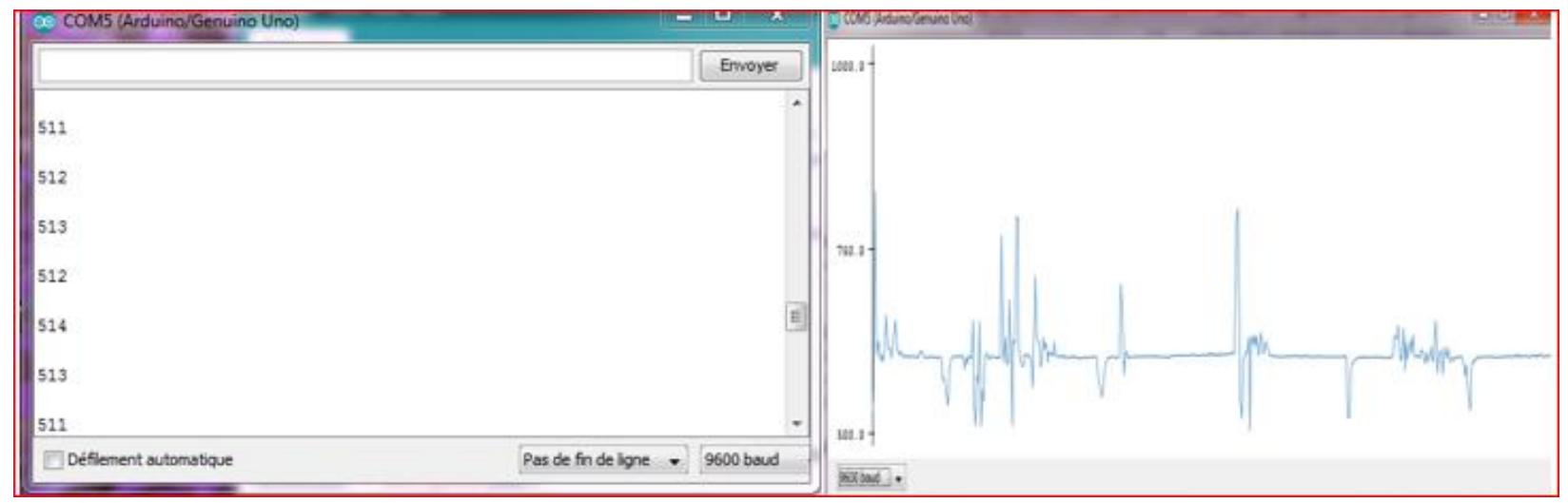

Figure 3. Visualization of the data on the serial monitor and serial plotter of the IDE. 
The data displayed on the serial monitor is saved to a file, and the graphical interface program is started to allow the EMG signal to be mapped.

\section{EMG signal processing and analysis:}

The EMG signal once acquired is processed and analyzed. Digital processing is particularly relevant to spectral analysis and envelope detection, but the analysis concerns the evaluation of the spectral content and the envelope respectively for different EMG cases depending on the size and age of the subject. The intensity of the effort applied. However, and for a comprehensible representation of the EMG signal on the graphical interface (i.e. amplitude in volts and duration depending on time), normalization step has been developed to convert the values of the samples into volts and their variation in depending on the sampling frequency in time.

\subsection{Normalization:}

The (Figure 4) below describes the EMG signal collecting activity on the muscle of an exerting arm. It is a signal drawn directly by using the samples acquired through the acquisition card (after offset circuit) at the sampling frequency. It is considered as non-standardized EMG signal. For a more understandable representation this signal is normalized through the routine described below.

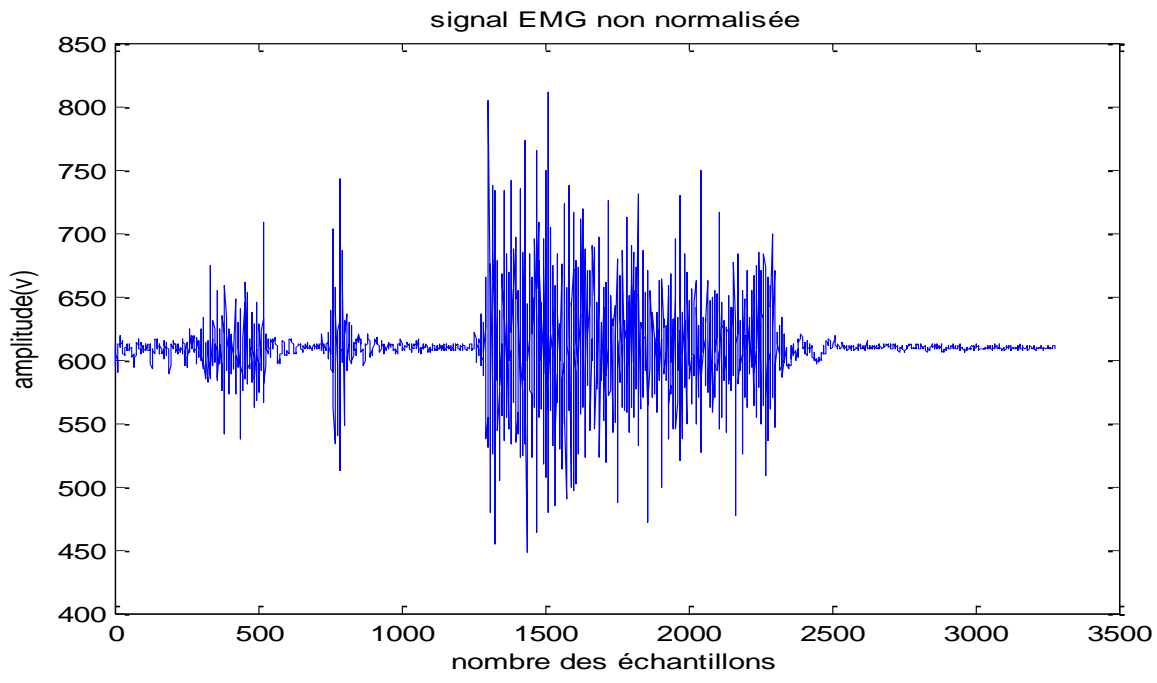

Figure 4. EMG signal not standardized.

To normalize our signal it is necessary to go through three operations:

$\checkmark \quad$ Divide the amplitudes of the signal by the gain introduced at the circuit during the detection

$\checkmark \quad$ Divide the signal length by the sampling frequency so that the abscissa axis can be adapted as a function of time.

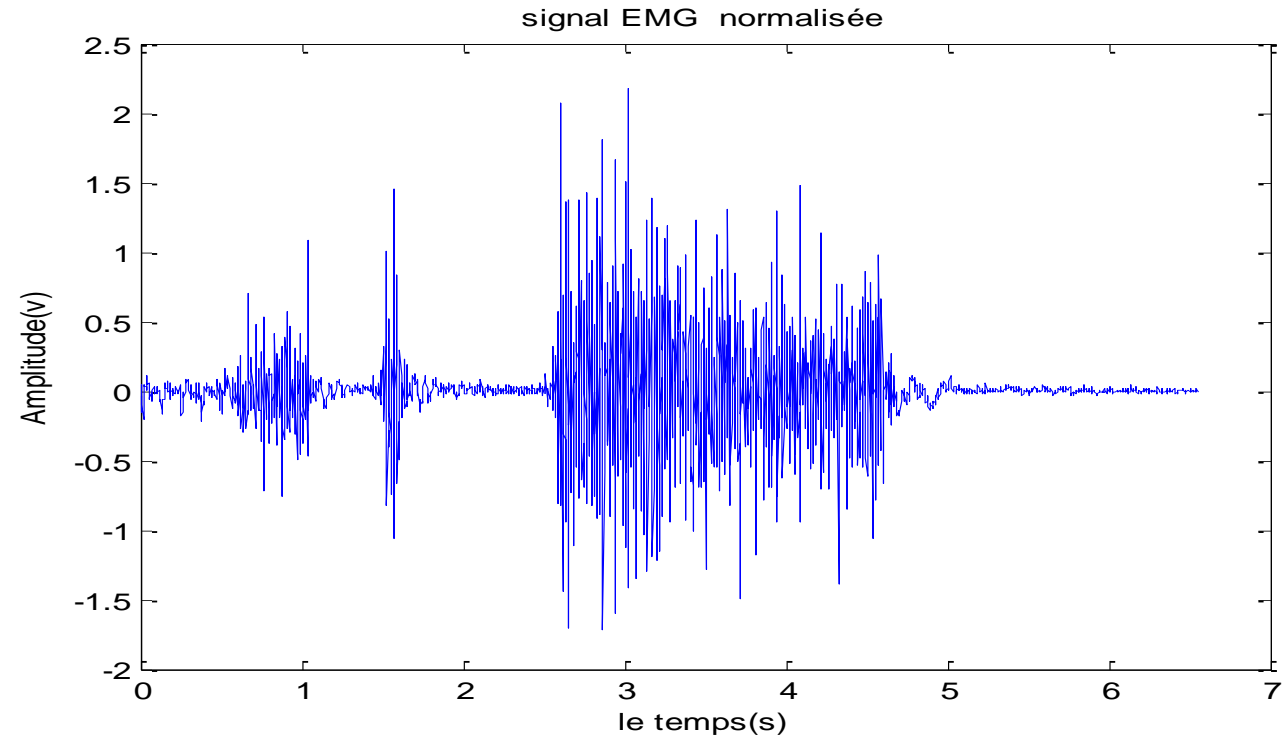




\subsection{EMG signal envelope detection:}

In order to evaluate the intensity of the effort through the EMG signal, the signal envelope is detected. This is detected through the development of a Matlab environment routine. This routine first allows a correction of the signal (rectification) then a low pass filtering with a cut off frequency suitably chosen [6]. In fact, the choice of the cutoff frequency depends on the high frequencies present in the rectified signal. On this subject, after rectification different cutoff frequencies of the low pass filter (the low pass filter chosen in our case is the Butterworth filter) [7] were tested to finally choose the most appropriate frequency.
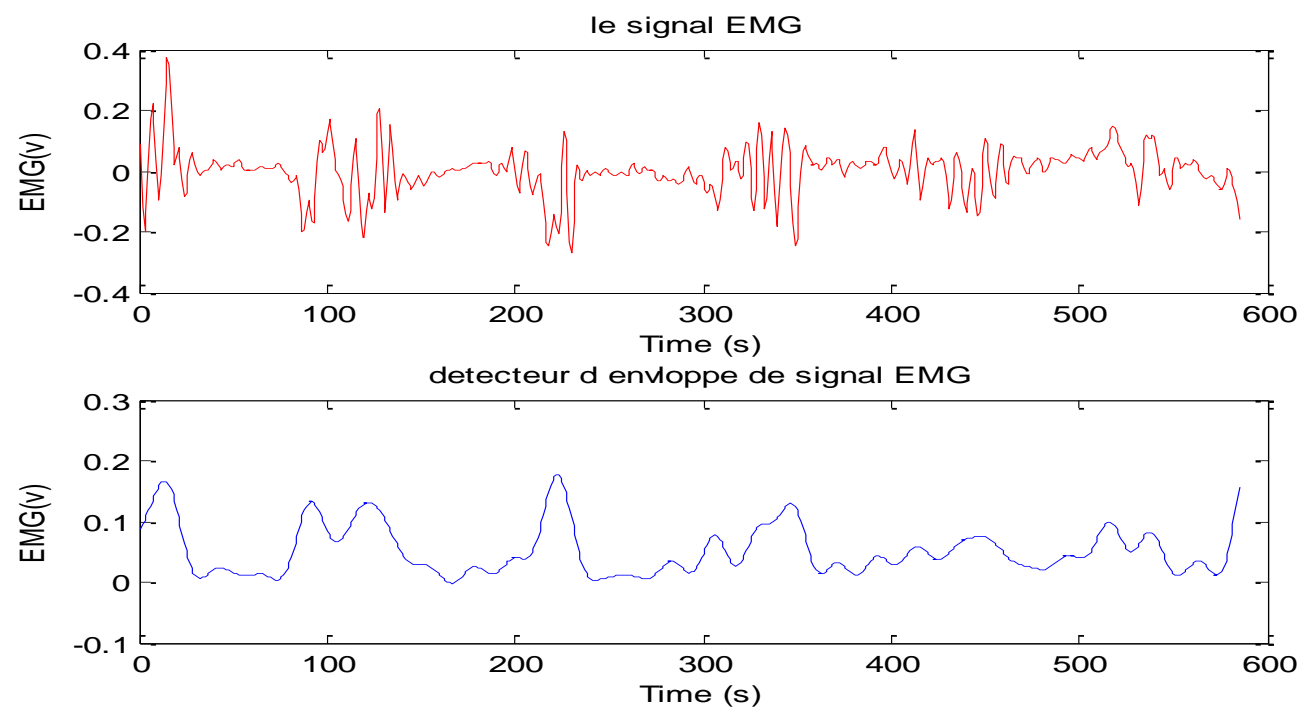

Figure 6. Plotting of the EMG signal and its envelope obtained with a low-pass filter of $40 \mathrm{~Hz}$ cut-off frequency

From the cutoff frequency test results, it is clear that low pass filter with the cutoff frequency of $40 \mathrm{~Hz}$, generates the most significant envelope. However, for the case of the $10 \mathrm{~Hz}$ and $20 \mathrm{~Hz}$ cutoff frequencies, the detection is very vague, and for $100 \mathrm{~Hz}$ the generated signal is similar to the original signal. The filter with the cutoff frequency of $40 \mathrm{~Hz}$ is then used for further analysis of the effort.

\subsection{Spectral analysis:}

In order to evaluate the variation of frequency content as a function of the effort. It returns to make a spectral analysis of a signal that identifies each of these frequency components and their respective importance. What is called "importance" is the amount of energy (or power) that each frequency component carries.

Fourier analysis is one way to do this analysis. It allows a signal to be broken down into a sum of particular elementary (sinusoidal) signals with adequate coefficients. It makes it possible to associate with the usual " waveform " the complementary representation in the frequency domain. This decomposition is obtained on the discrete signals through the Discrete Fourier transform TFD. It is obtained through a known calculation algorithm by fast Fourier transform (FFT). This is a particular DFT calculation algorithm. It is essentially based on the following constraint: the number of samples is imposed to be of the form $2^{i}$.

$$
\mathrm{N}=2^{\mathrm{i}}
$$

We know that the calculation of DFT is associated with the calculation of the following sum:

$$
\sigma \mathrm{k}=\sum_{n=0}^{N-1} \operatorname{Sn} e^{\frac{j k 2 n}{N}}
$$

This computation has $\mathrm{N}$ multiplications between $\mathrm{Sn}$ and complex exponentials, and $\mathrm{N}$ additions in total; the calculation of the most penalizing multiplications in term of duration. However, the imposed constraint makes it possible to simplify and accelerate their calculation.

\section{Application of the FFT(Fast Fourier Transform) on the EMG signal}

The FFT is applied to the EMG signals in order to analyze the spectral content to assess the effort with respect to the frequency content [9].

\section{Measurements of muscular effort:}

In this part we made spectral analysis on seven participants to compare the frequency content between them in the cases: rest, average and with a strong muscular effort, the following (Table 1) describes the information of each participant (age, height, weight, sex).

\begin{tabular}{|l|c|c|c|c|}
\hline Les sujets & Age (years) & Height(m) & Weight(Kg) & Sex \\
\hline Participant1 & 15 & 1.60 & 41 & Man \\
\hline Participant 2 & 11 & 1.3 & 35 & Woman \\
\hline Participant 3 & 23 & 1.69 & 55 & Woman \\
\hline Participant4 & 5 & 0.6 & 19 & Woman \\
\hline Participant 5 & 45 & 1.64 & 78 & Man \\
\hline Participant 6 & 24 & 1.70 & 70 & Man \\
\hline Participant 7 & 24 & 1.60 & 45 & Woman \\
\hline
\end{tabular}

Table 1. The information of each participant 
We measured the EMG signal of each in three cases, the first at rest (the volunteer releases his arm), then a contraction lasting a few seconds or a half seconds, after a slow or rapid effort also lasting a few seconds.
This experiment on young 15 years where he made the three cases at the same time, we detected the envelope of its signal EMG then the spectral analysis.

\section{Participant 1:}
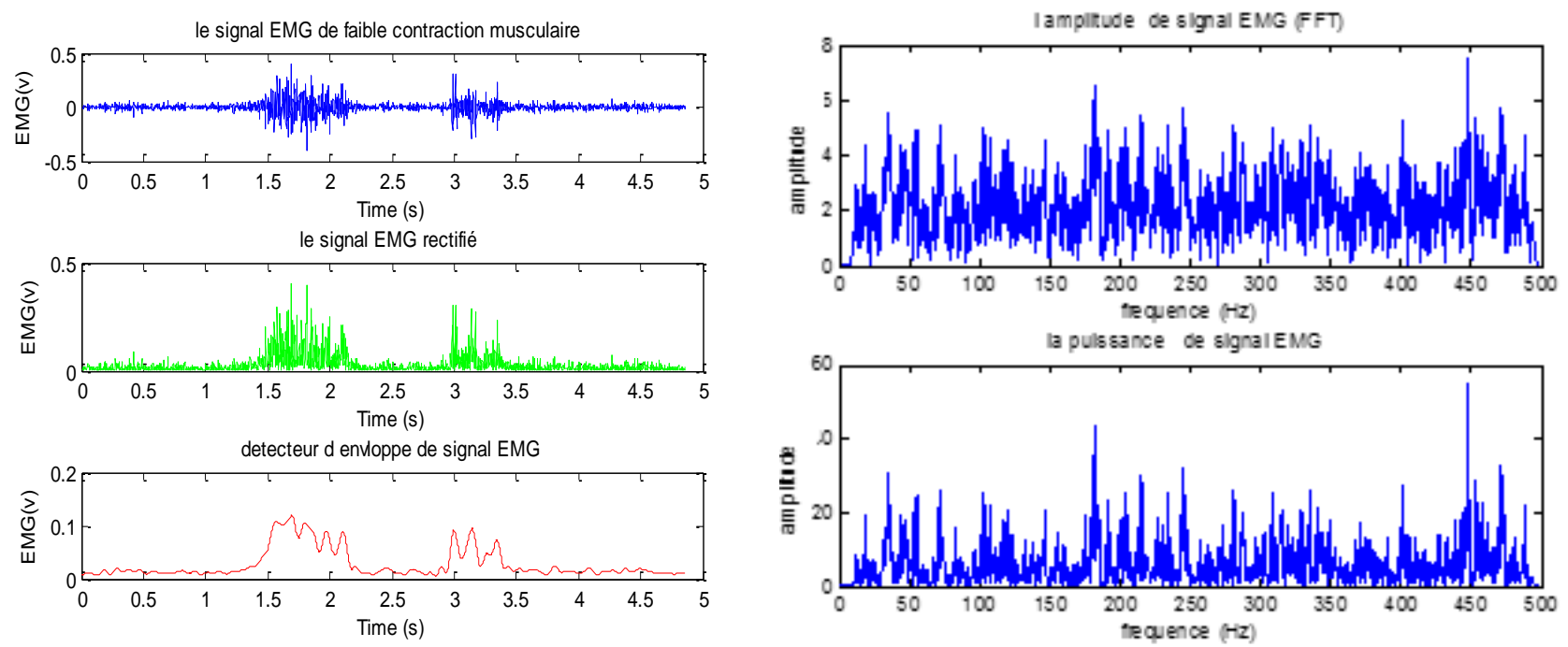

Figure 7. The EMG signal of a volunteer, the envelope and frequency content.

When the subject makes several contractions and especially of different muscular level at the same time, we cannot extract the frequency content of the whole signal so we take just one contraction each time is the purpose of this experiment.

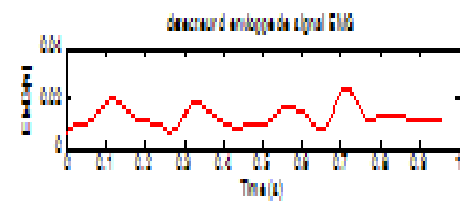

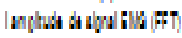
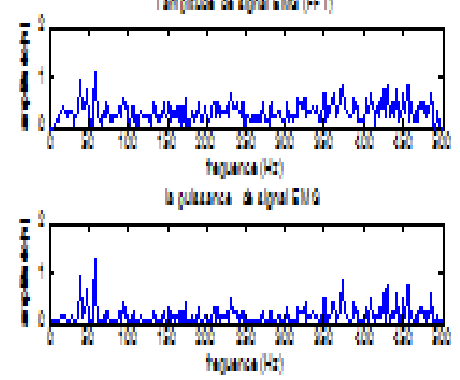

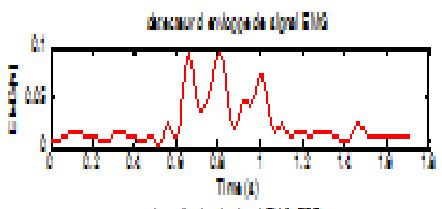

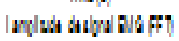

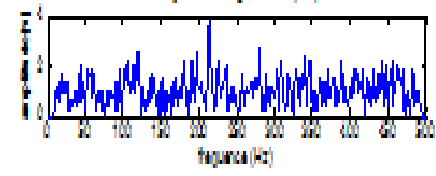

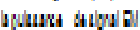

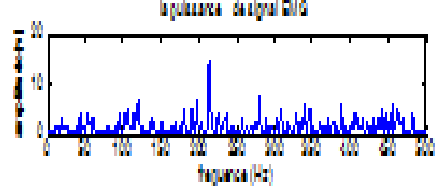

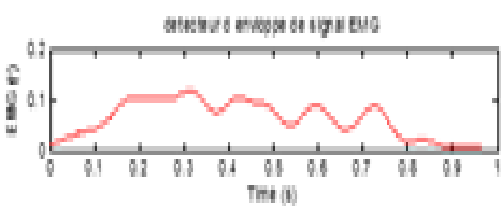

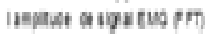

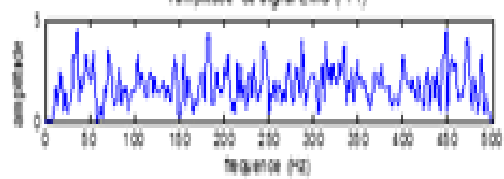

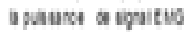

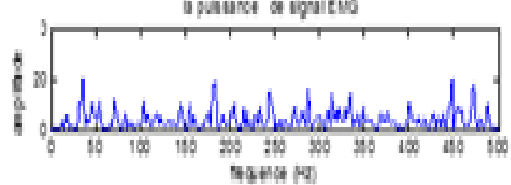

Figure 8. The envelope and frequency content of EMG signal of subject 1

\section{Participant 2:}
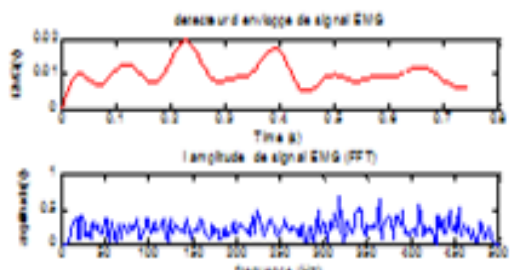

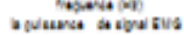

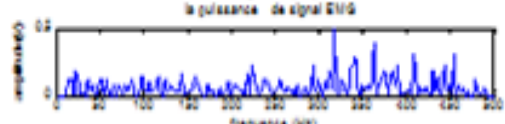

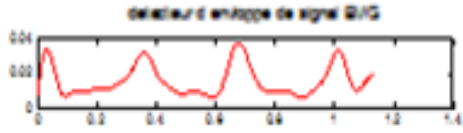

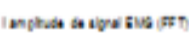

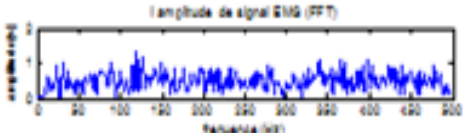

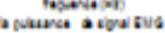

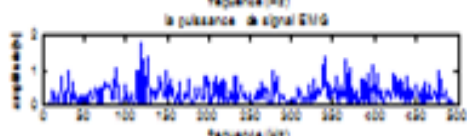

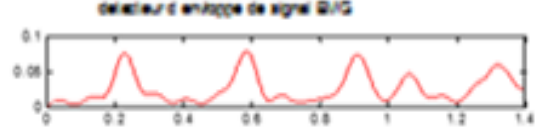

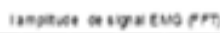

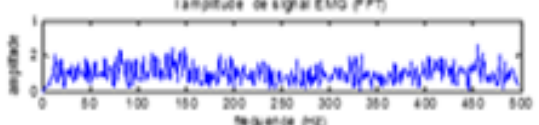

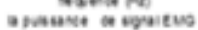

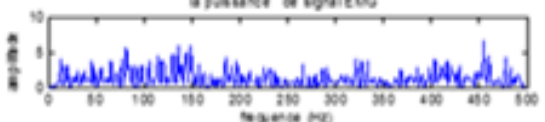

Figure 9. The envelope and frequency content of EMG signal of subject 2 


\section{Participant 3 :}
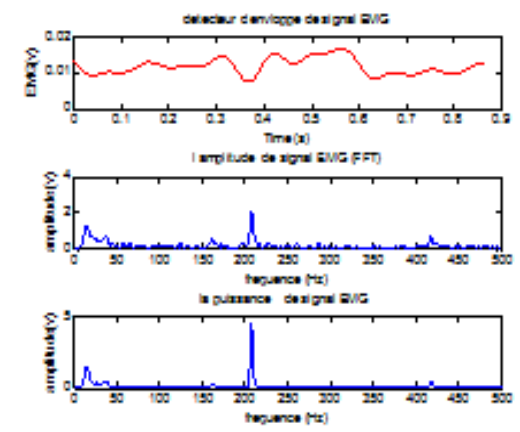

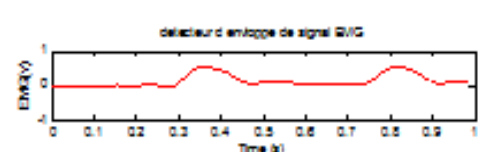

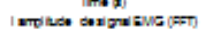

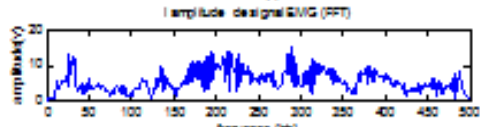

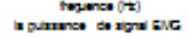

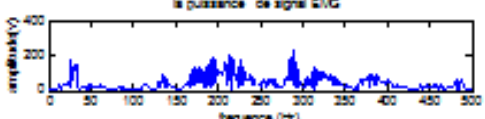

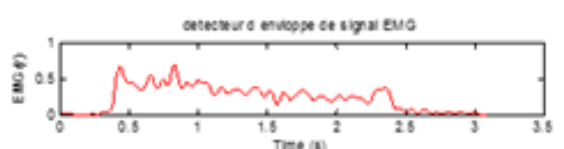
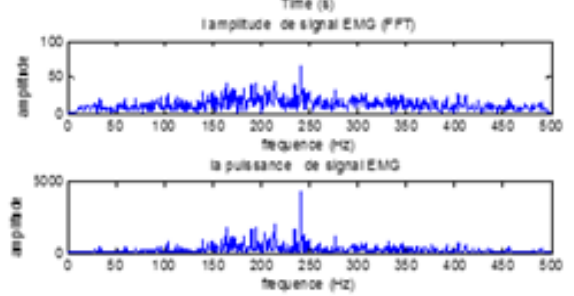

Figure 10. The envelope and frequency content of EMG signal of subject 3

\section{Participant 4:}

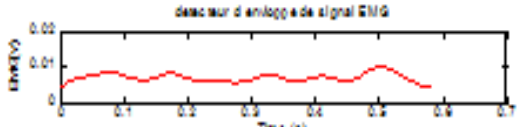

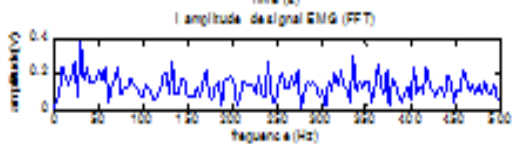

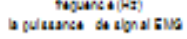

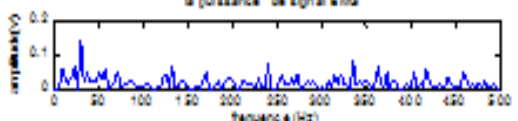

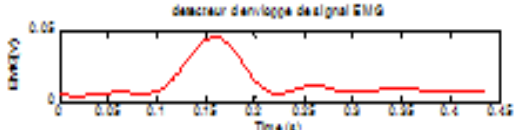

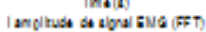

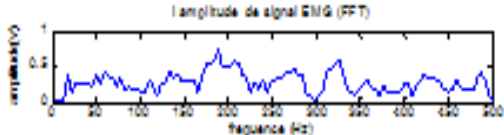

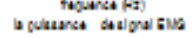

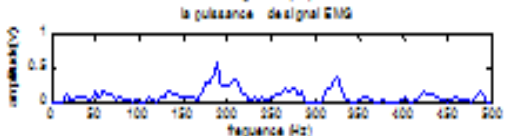

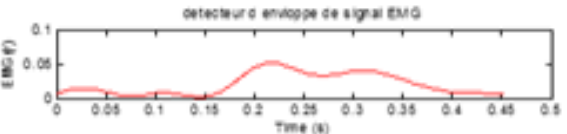

Iampance of tigu two cm?

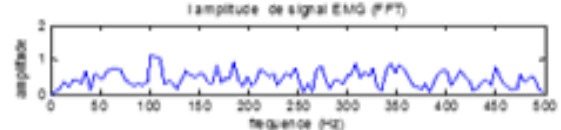

opuases of arvieng

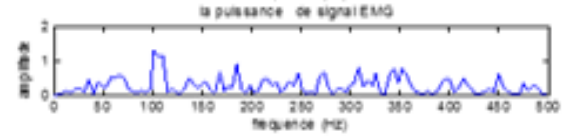

\section{Figure 11. The envelope and frequency content of EMG signal of subject 4}

\section{Participant 5:}

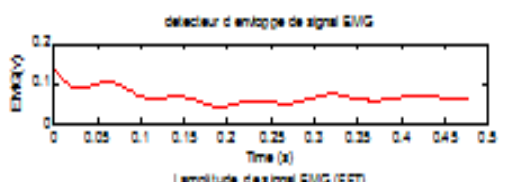

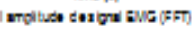

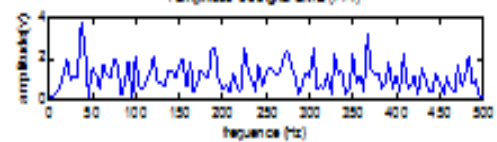

la pimanes de rove ave

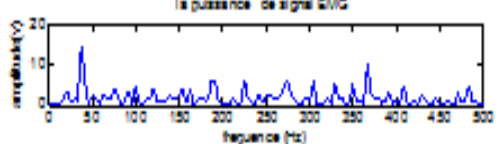

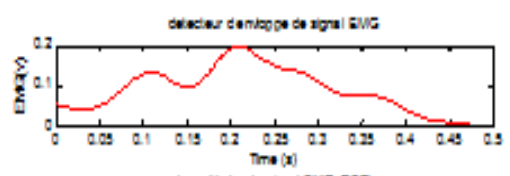

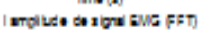

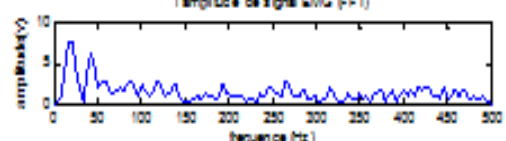

a gumence dongu Bue

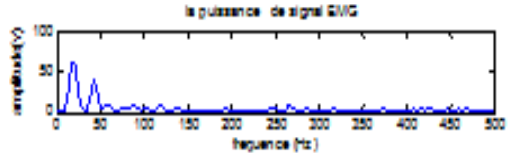

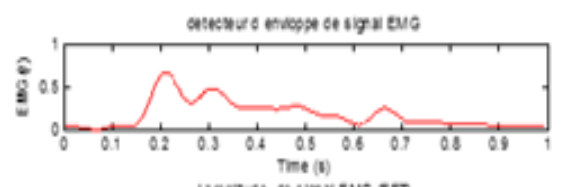

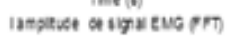
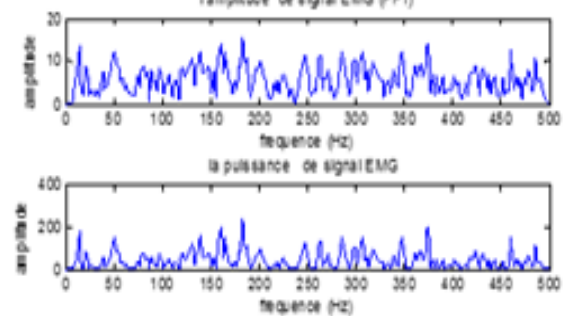

Figure 12. The envelope and frequency content of EMG signal of subject 5

\section{Participant 6:}

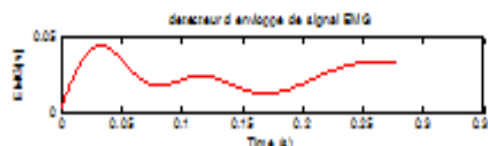

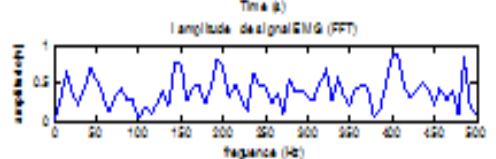
bouanse do arou ana

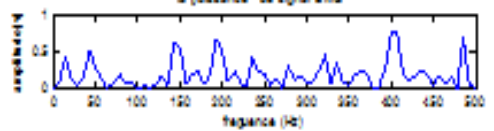

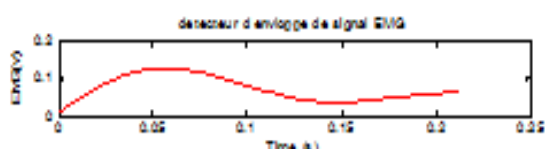

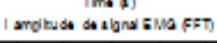

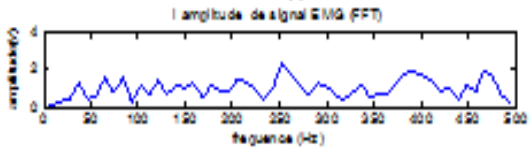

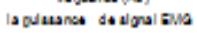

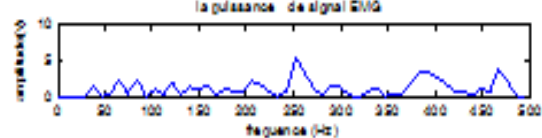

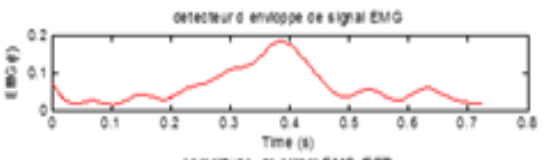

umpatise of igx eno $\mathrm{rm}$

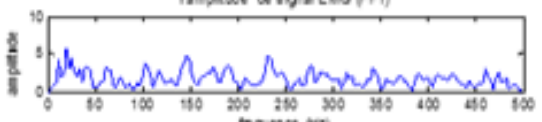

a pauste on egraicug

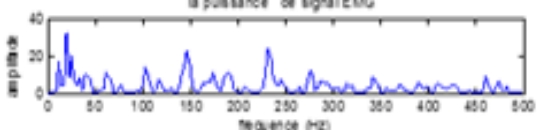

Figure 13. The envelope and frequency content of EMG signal of subject 6 


\section{Participant 7 :}

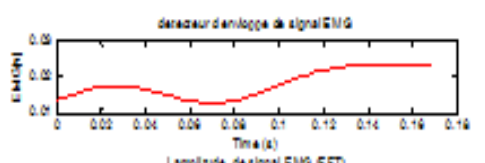

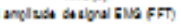

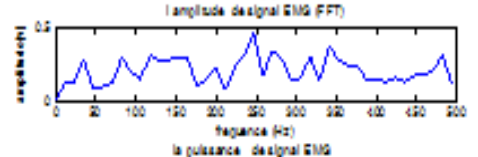

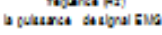

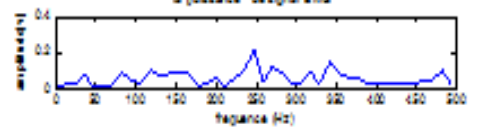

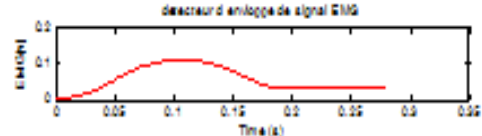

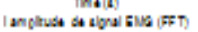

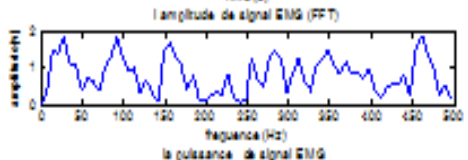

Lo guanse a doral ane

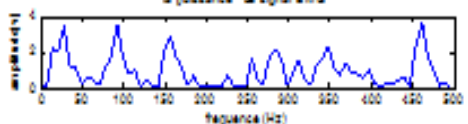

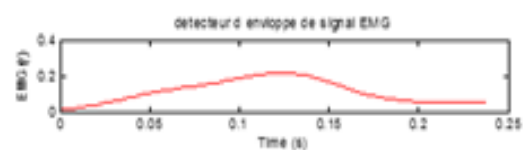

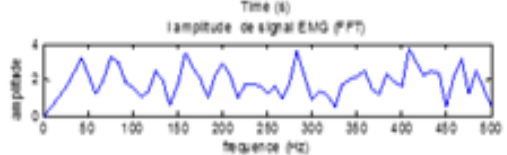

apuatast of agaicug

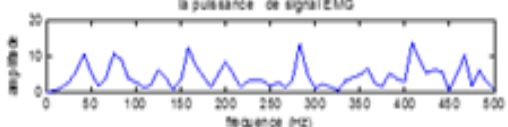

Figure 14. The envelope and frequency content of EMG signal of subject 7

\section{Result and Discussion:}

In our project, seven treated subjects participated in the study to make measurements after having informed consent about the safe procedure associated with the study. They were instructed to perform voluntary contractions of the biceps. Surface EMG signals were collected from the arm muscles. The power of the signals (from the spectrum) was then analyzed to evaluate the effort by taking into account physical variations such as height and weight, and it was noted that the latter directly influenced muscular effort.

A power spectral analysis was performed to determine the characteristics of the EMG in the muscles concerned to evaluate the muscular effort in order to examine the relationship between the spectral parameters and the contraction levels.

What we can notice is the specificity is due to the variations existing between the individuals, among which we find the physical variations (ex: the size, the weight, the corpulence).

According to the individuals, the same information does not indicate the same interpretation.

\section{$>$ The envelope detector}

The representation of the signal envelope allows a pleasant visualization of the periods of activation of the muscle.

- $\quad$ For a weak effort we notice that:

- Three subjects among them their amplitude can wait up to $0.02 \mathrm{v}$ and 2 subjects their amplitude can reach only $0.01 \mathrm{v}$ it is because of the age for the subject 5 or of corpulence of the subject 4 .

- $\quad$ On the contrary for the seventh subject, young of 24 years their amplitude reaches up to $0.05 \mathrm{v}$

- $\quad$ For a lot more effort, the amplitude will be multiplied again.

- $\quad$ For a strong effort we note that:

During a very strong effort the seven subjects presents the same reponse that is to say the intensity of the signal increases.

As muscular contraction intensity increases, each UM is recruited at a higher frequency and the number of UMs recruited also increases, which leads to an enrichment in PUMs of the recordings.

$>\quad$ Signal amplitude (FFT):

- $\quad$ Spectral lines appear more and more with effort. .

- As the effort increases, we notice that all the subjects have more stripes.

- $\quad$ For a strong effort we note that:

These lines will be reinforced with a much more remarkable the frequency content of the EMG signal changes according to a variable set such as the physical variations of the subject (age, height, weight ...), good analog and digital filtering, the health of the subject, the right way to make a contraction and the good EMG signal acquisition.

Through the envelope we can appreciate the effort. Indeed the amplitude of this envelope reflects the intensity of the effort, and its duration the duration of the effort.

The muscular effort is also analyzed through the Fourier transform (spectral analysis) to determine the frequency content and therefore evaluate its variation as a function of the effort.

It can clearly be noted that the envelope detection and the analysis of the frequency content of the EMG signal allowed us to evaluate the muscular effort.

\section{Conclusion}

In this work, we are interested in the practical theoretical study and realization of a device for the evaluation of the muscular effort through the signal Electromyogram EMG.

The approach that has been adopted for the realization of this project is that used by an engineer in the design, development and realization; namely to establish a specifications, see and study the different circuits that can be used, and finally the practical realization.

Thus, first a bibliographic research study was conducted. Through this study, the Electromygraphic signal has been described from its source at the cellular level to its appearance on the surface of the skin. This study was followed by a theoretical study of the circuits that can be used to detect, format and acquire the EMG signal. Thus, theoretical notions about sensors, instrumentation amplifier, filtering and acquisition have been recalled. The manuscript was finalized by our own contribution namely the practical realization of the device allowing the evaluation of the muscular effort through the EMG.

Thus, the device made enables the detection of the EMG signal, its shaping, and its acquisition through an ARDUINO acquisition card to a PC, and its digital processing to evaluate the muscular effort. This digital processing was mainly frequency analysis and envelope detection, or the results obtained showed that the frequency content as well as the signal envelope changed according to the effort. These results as well as the EMG signal are displayed on a graphical interface that has also been developed in this work.

\section{References}

1. Lori Newell (2017). “A Description of Tendons, Ligaments and Muscles" Partner \& Licensee of the Livestrong Foundation.

effort. 
2. Peter Konrad (2006). "A Practical Introduction to Kinesiological Electromyography” Noraxon INC. USA.

3. Kassou Otmane and Rahmani Youssouf (2015). "Study and Realization of an Emg Signal Acquisition System by Bluetooth" University of Tlemcen.

4. Tamara Grujic Supuk, Ana Kuzmanic Skelin, \& Maja Cic Design, (2014). "Development and Testing of a Low-Costs EMG System and Its Use in Recording Muscle Activity in Human Gait" National Center for Biotechnology Information, U.S. National Library of Medicine.

5. Louis Reynier. (2010). "What is Arduino?" Electronics in BTS of Electronic Systems at the Cabanis high school in Brive-laGaillarde.

6. Mariaconcetta Vinti. (2012). "Biomechanical and Physiological Characterization of Spastic Cocontraction in Spastic Paresia"
Arts and Crafts Paris Tech - Paris Center Biomechanics Laboratory.

7. Dal Maso Fabien. (2012). "Involvement of the primary motor cortex in the regulation of muscle coactivation. Study of the modulation of cortical oscillations and cortico-muscular interactions" University of Toulouse.

8. A. Rabbahi \& I. Oukili (2015). "Realization and acquisition of the EMG circuit with the arduino card in order to send it to smartphones" Ain Témouchent university center.

9. M.B.I. Raez, M.S. Hussain, \& F. Mohd-Yasin (2006). "Techniques of EMG signal analysis: detection, processing, classification and applications" National Center for Biotechnology Information, U.S.
This work is licensed under Creative Commons Attribution 4.0 License

To Submit Your Article Click Here: Submit Manuscript

DOI: $10.31579 / 2690-1919 / 169$
Ready to submit your research? Choose Auctores and benefit from:

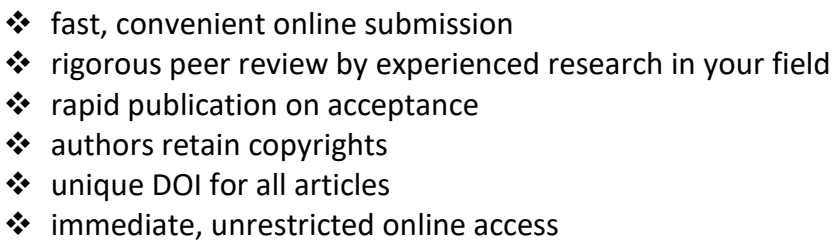

At Auctores, research is always in progress.

Learn more www.auctoresonline.org/journals/journal-of-clinicalresearch-and-reports- 